\title{
TOPONIMIA DOCUMENTAL DEL VALLE MEDIO DEL ÉSERA, III: TOPONIMIA DEL AYUNTAMIENTO DE FORADADA DEL TOSCAR (HUESCAR) ${ }^{1}$
}

MOISÉs Selfa SASTRE

Universidad de Lérida

\section{RESUMEN}

En este artículo se analiza lingüísticamente la toponimia documental que pertenece a los núcleos de población del Ayuntamiento de Foradada del Toscar. Para ello partimos, principalmente, de la lectura dos fuentes documentales inéditas: los Amillaramientos con rectificaciones hasta el año 1879 (Am.) conservados en el Archivo Histórico Provincial de Huesca (AHPH) y, sobre todo, los Protocolos Notariales de Pedro de Guart (Protocolos) conservados en el Archivo Capitular de Lérida (ACL). El análisis se realiza desde el punto de vista tradicional de la toponimia diacrónica. La mayoría de los topónimos estudiados proceden de una base lingüística latina; otros, en cambio, están relacionados con las lenguas prerromanas (barza, clot, coma, por ejemplo) y con el elemento lingüístico vasco (Gabessaco).

1 Este trabajo se enmarca dentro del proyecto de investigacion Toponimia de Ribagorza coordinado desde 1995 por el Dr. JAVIER TeRrADo (Universidad de Lérida) y financiado por el Ministerio de Educación y Cultura (PS94-0208), la Generalitat de Catalunya y el Ayuntamiento de Lérida.

${ }^{2}$ Quisiera agradecer las jugosas observaciones que a este trabajo me realizaron los doctores Ramon Sistac i Vicén (Universidad de Lérida), Javier Terrado Pablo (Universidad de Lérida) y Jesús Vázquez Obrador (Universidad de Zaragoza. Campus de Huesca). 


\section{INTRODUCCION ${ }^{2}$}

Continuamos con este artículo el estudio de la toponimia documental de la zona geográfica oscense del Valle Medio del Ésera, que fue iniciado con otros dedicados a los macrotopónimos y microtopónimos de este amplio sector geográfico.

En esta ocasión se recogen los materiales toponomásticos que pertenecen al Ayuntamiento de Foradada del Toscar: Bacamorta, Espluga, Foradada del Toscar, Las Colladas, Navarri, Senz, Valle de Terraza y Viu. Se exceptúa la toponimia documental de Murillo de Liena que fue objeto del segundo artículo de los que consta esta serie. El fin que más nos ha interesado ha sido el de aportar y rescatar del olvido toda una serie de nombres, tanto de lugar como apelativos, que todavía están vivos entre las gentes más ancianas del pueblo. En este sentido, la lectura de las fuentes documentales juega un papel esencial.

Para la organización, y de acuerdo con los esquemas ya clásicos, se han establecido diversos apartados, según áreas de pertenencia de los topónimos. La estructura que seguimos para el análisis e interpretación de cada topónimo en sí es la siguiente:

a) Forma documental. Cuando se trata de un topónimo compuesto, indicamos entre parentesis la parte del mismo que se estudia en otros apartados. Por ejemplo, en el campo semántico de los zootopónimos, en un topónimo como «Abelas (abellanero), lo campo de las», como sólo nos interesa estudiar «Abelas» y no "abellanero», entre paréntesis presentaremos esta última parte del topónimo: «Abelas (abellanero), lo campo de las». Si el topónimo pervive en la actualidad lo indicamos con el signo de «+». Si, por el contrario, se trata de un topónimo desconocido lo indicamos mediante el signo «-». Por otro lado, es necesario advertir que si un topónimo presenta dos o más variantes, la explicación etimológica sólo se dará en la primera de sus entradas léxicas. Por ejemplo, véase la serie del topónimo Torre que estudiamos en el apartado de las construcciones humanas.

b) Si el topónimo es reconocido por las gentes a las que realizamos la encuesta, indicamos su pronunciación popular;

c) a continuación, presentamos el contexto lingüístico en que se documenta el topónimo que estamos analizando;

d) por último, indicamos su etimología. Normalmente, tras indicar el étimo y su significado, remitimos directamente a las explicaciones que nos ofrecen el DCECH, el DECat o el OnCat. Además, siempre que es posible, ofrecemos el significado de la voz, si conserva su valor apelativo, así como su pronunciación popular. 
CORPUS TOPONIMICO

\section{Construcciones humanas}

\subsection{Ostalet, el - Valle de Terraza}

Documentación:

a. 1624. «die undeçimo mensis augusti anno 1624 en el ostalet...» (ACL, Protocolos, vol. $n .^{\circ} 14, f .91 v$ ).

Etimologia: topónimo derivado mediante el sufijo -rTTU (>-et) del apelativo latino HOSPITALE «el que ospeda» (DCECH, s.v. huésped). Se trata de un vocablo que designa un conjunto de casas prácticamente deshabitadas, pero que antaño tuvieron su importancia como lugar de acogida de los viajeros que cruzaban a pie la red de caminos que comunica el Valle del Isábena con el del Ésera.

\subsection{Quadras, las ceresseras de las - Senz}

Documentación:

a. 1613. «...sitio en el termino de dicho lugar de Seira en la partida llamada a las ceresseras de las quadras...» (ACL, Protocolos, vol. $n .^{\circ} 4$, f.79v).

Etimología: del latín QUADRUM «un cuadrado» (DCECH, s.v. cuadro). Apelativo que refiere «una casita o cabaña de pastor en la montaña, que pertenece generalmente a una masía de la zona»; [lakwádra].

\subsection{Torre, campo la + Bacamorta}

\section{Pronunciación: [lokámpodelatóre $]$}

Documentación:

a. 1614. «...en el termino del lugar de Bacamorta y Valle de Terraça en la partida llamada a campo la torre...» (ACL, Protocolos, vol. $n .^{\circ} 5, f .85 v$ ).

Etimología: del latín TURRIS «torre, torreón» (DCECH, s.v. torre). Voz de uso general en todas las épocas y común a todos los romances. En nuestra área de estudio es un sustantivo sinónimo de castell «construcción en la que se guardan los instrumentos agrícolas». En cuanto a la elisión de la preposición de, ésta se explica por fonética sintáctica: [kámpodelatóre > kámpo'elatórè kámpolatóre]. 


\subsection{Torre, cassas de la - Valle de Terraza}

Documentación:

a. 1609. «...un campo de la cassa de miguel de la casta de las cassas de la torre...» (ACL, Protocolos, vol. $n .^{\circ} 1, f .150 r$ ).

Etimología: véase s.v. Torre, campo la. Conjunto de casas que no hemos conseguido localizar en la toponimia viva del Valle de Terraza.

\section{FITOTOPONIMOS}

\subsection{Abellanero, campo de las abelas - Foradada del Toscar}

Documentación:

a. 1610. «...item otro campo sitio en el mesmo termino de Foradada en la partida llamada a lo campo de las abelas abellanero..." (ACL, Protocolos, vol. $n .^{\circ} 2, f .180 r$ ).

Etimología: topónimo derivado en -ARIU (>-ero) del latín ABELLANA «avellana», forma abreviada de ABELLANA NUX (DCECH, s.v. avellana). Voz de uso común en toda nuestra zona de estudio; [labelanéro].

\subsection{Barza, plana la + Bacamorta}

Pronunciación: [laplánadelabár $\theta a]$

Documentación:

a. 1609. «...en el termino del lugar de bacamorta en la partida llamada a plana la barza...» (ACL, Protocolos, vol. $n .^{\circ} 1, f .194 r$ ).

Etimología: apelativo de origen incierto para el que Coromines (DECat III, s.v. esbarzer, $437 \mathrm{~b} 11 \mathrm{ss}$ ) propone un étimo en todo caso prerromano y seguramente no indoeuropeo; probablemente esté emparentado con el gascón barta «conjunto de plantas espinosas» (<*BARTIA). La terminación $-z a$ de nuestra forma parece ser que es la misma del vasco-ibérico «-tza» de carácter colectivo.

Barza es una voz de uso general en nuestra zona de estudio: se refiere, como en gascón, a aquel «conjunto de plantas espinosas que muchas veces son utilizadas para rodear los establos en donde se guarda el ganado».

\subsection{Binia (de la cassa de Gabessaco), la - Foradada del Toscar}

Documentación:

a.1612. «...confronta con binia de la cassa de gabessaco...» (ACL, Protocolos, vol. $n .^{\circ} 3, f .127 r$ ). 
Etimología: del latín VĪNĚA ( $D C E C H$, s.v. vino). Voz de uso general en todos las épocas y común a todos los romances hispánicos. Normalmente, este apelativo toponímico indica «pequeños trozos de terreno cercanos al río".

\subsection{Binia (de lo puzol), la - Bacamorta}

\section{Documentación:}

a. 1609. «...et asi ajuntado el dicho consejo en la binia de lo puzol...» (ACL, Protocolos, vol. $n .{ }^{\circ} 1, f .64 r$ ).

Etimología: véase s.v. Binia (de la cassa de Gabessaco), la.

\subsection{Ceresseras (de las quadras), las - Senz}

Documentación:

a. 1613. «...sitio en el termino de dicho lugar de Seira en la partida llamada a las ceresseras de las quadras..." (ACL, Protocolos, vol. $n .^{\circ} 4, f$. $79 v)$.

Etimología: Ceresa, del latín vulgar CERĚSIA «cereza» (DECat II, s.v. cirera, $713 \mathrm{~b} 35 \mathrm{ss}$ ), adquiere el significado general de «árbol cuyos frutos son las cerezas». Esto se debe a que en latín vulgar cerasia fue utilizado no sólo como nombre del fruto, sino también, al igual que cesarium, como nombre del árbol. En cuanto al sufijo -era (<-ARİ) forma nombre de plantas y árboles.

\subsection{Espinalba, la - Bacamorta}

\section{Documentación:}

a. 1623. «...en el termino del lugar de Bacamorta en la partida llamada a la espinalba...» (ACL, Protocolos, vol. $n .^{\circ} 13, f .203 r$ ).

Etimología: el apelativo espinablo «majuelo» es hoy poco usado. Proviene mediante metátesis de la líquida de espinalbo, vocablo compuesto por espino (derivado del latín SPİNA «espina vegetal») (DCECH, s.v. espina) y albo «blanco» (DCECH, s.v. albo) (VÁZQUEZ, 1994:218).

Espinalba es un topónimo desconocido de la toponimia viva de Bacamorta, por lo que creemos que se trata del femenino de espinalbo, $-a<e s-$ pinablo. 


\subsection{Guncar, lo campo de lo + Bacamorta}

Pronunciación: [lokámpodelĉunkár]

Documentación:

a. 1609. «...en la partida llamada a lo campo de lo guncar...» (ACL, Protocolos, vol. $1, f .106 r)$.

Etimología: topónimo colectivo derivado en -ĀRE sobre el latín JŬNCUS «junco» (DCECH, s.v. junco) con la evolución típicamente altoaragonesa $\left.J_{-}\right\rangle[\hat{c}]\langle g\rangle$. Voz que se refiere a aquellos «trozos de tierras próximos a parajes húmedos en los que que generalmente abundan los juncos».

\subsection{Lapacil, coma- - Espluga}

Documentación:

a. 1608. «...un campo mio sitiado en el termino despluga en la partida llamada comalapacil...» (ACL, Protocolos, vol. $n .^{\circ} 1, f .42 v$ ).

Etimología: del latín LAPPACEUS «perteneciente al lampazo» (DCECH, s.v. lampazo) al que se le añade el sufijo locativo -ĪLE (>-il) (GIRALT, 1995:241). Se trata éste de un apelativo que indica «un campo de cultivo en cuyos márgenes se crían un tipo de hierbas perennes cuyos pétalos son de color purpúreo». Como voz de uso, [lalapá日a], susbsiste por lo general en todo el Valle Medio del Ésera junto a bardana [labardána].

\subsection{Llezina, campo la + Viu}

\section{Pronunciación: [lokámpođeląle Bína]}

Documentación:

a. 1613. «...en el dicho termino del dicho lugar de Viu en la partida llamada a campo la llezina...» (ACL, Protocolos, vol. $n .^{\circ} 4, f .219 \mathrm{v}$ ).

a. 1879. «la lecinera» (AHPH, Am. 1072, cuad. $\left.1 .^{\circ}, f .67 r\right)$

Etimología: del latín vulgar ILĪCINA, derivado adjetivo de İLEX, İLICIS «encina» (DECat, s.v. alzina). Llecina es una voz común al aragonés que, según nuestros informantes, se refiere a aquel «campo en el que abundan este tipo de árboles y que normalmente se utiliza para el pastoreo del ganado». 


\subsection{Salitar, lo + Navarri}

\section{Pronunciación: [losalitár]}

Documentación:

a. 1624. «...item otro campo que nuestro que propio que tenemos sitio en el mismo termino y partida de lo salitar...» (ACL, Protocolos, vol. $n .^{\circ} 14, f .2 r$ )

a. 1879. «salitar» (AHPH, Am. 1072, cuad. 1. ${ }^{\circ}$ f. 90r)

Etimología: del latín SALICTUM, a su vez de SALIX, -ICIS «sauce» (DCECH, s.v. sauce) al que se le añade el sufijo locativo -ĀRE. Voz común en toda nuestra zona de estudio que designa aquel «conjunto de pequeños árboles de ribera».

\subsection{Serbera (castelaes) - Valle de Terraza}

\section{Documentación:}

a.1609. «...en el termino de la Balle de Terraza en la partida llamada a serbera castelaes...» (ACL, Protocolos, vol. $n .^{\circ} 1, f .196 r$ )

Etimología: topónimo sufijado en -era (<-ARIA) de serba (<SORBA, plural de SORBUM «fruto del serbal») (DCECH, s.v. serba). Voz común en el habla de nuestra zona de estudio que apela aquel «árbol de gran altura cuyas flores son de color blanco»; [laserbéra].

\section{Formas DE RELIEVE}

\subsection{Clot (de los guertos), lo + Navarri}

\section{Pronunciación: [loklót]}

Documentación:

a.1625. «...en el dicho termino de Nabarri en la partida llamada a lo clot de los guertos...» (ACL, Protocolos, vol. n. $\left.{ }^{\circ} 15, f .10 v\right)$

1879. «clot» (AHPH, Am. 1072, cuad. $1^{\circ}, f .116 r$ )

Etimología: apelativo toponímico orográfico frecuente en nuestra zona de estudio. Su sentido es el de «pequeña valle» y corresponde a lo que en castellano denominaríamos vaguada (TERRADO, 1992:67). Para la etimología de la voz, véase lo que dice Joan Coromines en su DECat, s.v. clot, donde propone una base sorotáptica *KLŎPTON, neutro del participio *kloptós, que corresponde al griego $\kappa \lambda \varepsilon \pi \tau o \sigma$ «robado» y al prusiano antiguo au-klipts «escondido». De la idea de «escondrijo» se pasó a la de «concavidad que cubre». En 
la toponimia del Valle Medio del Ésera, clot indica una «depresión en la falda de una montaña».

\subsection{Coma, la $+\operatorname{Senz}$}

Pronunciación: [lakóma]

Documentación:

a.1609. «...a ssaber es un campo nuestro sitiado en el termino de dicha balle en la patida llamada a la coma...» (ACL, Protocolos, vol. $n .^{\circ} 1, f .163 v$ ).

a.1613. «...un campo mio proprio sitio en dicho termino de dicho llugar de Senz en la partida llamada a la coma...» (ACL, Protocolos, vol. $n .^{\circ} 4, f$. 223r).

a.1879. «la coma» (AHPH, Am. 1072, cuad. $\left.1 .^{\circ}, f .95 r\right)$.

Etimología: apelativo frecuente en nuetra zona de estudio así como en todo el dominio lingüístico catalán, gascón «couma» y provenzal «coumba». Su significado es el de «espacio de terreno llano entre otro accidentado» y supone una base *CUMBA relacionada con el celta CUMBOS «cuenca» (DECat 11 , s.v. coma, $849 a 41$ ss).

\subsection{Coma(lapacil) - Espluga}

Documentación:

a.1608. «...un campo mio sitiado en el termino despluga en la partida llamada comalapacil...» (ACL, Protocolos, vol. $n .^{\circ} 1, f .42 v$ ).

Etimología: véase s.v. coma, la.

\subsection{Panar (de las llagunas), lo + Foradada del Toscar}

\section{Pronunciación: [lopanárdelaslagúnas]}

Documentación:

a.1613. «...en el dicho termino de Foradada en la partida llamada a lo panar de las llagunas..." (ACL, Protocolos, vol. $n .^{\circ} 4, f .203 r$ ).

Etimología: apelativo sufijado en -ar que junto al mozárabe, castellano dialectal y portugués pando procede de PANDUS, -A, -UM «encorvado, convexo, cóncavo». Su sentido actual de «vertiente de una montaña más o menos elevada» es usual en toda la Alta Ribagorza e incluso en el Sobrarbe oriental. 


\subsection{Penna, la - Valle de Terraza}

Documentación:

a.1614. «...que confronta con la penna y con via publica...» (ACL, Protocolos, vol. n. ${ }^{\circ}$, f. $2 r$ ).

Etimología: del latín PĬNNA «almena». Su motivación semántica con el sentido actual de «piedra grande sin labrar» se debe a que las rocas que erizan la cresta de un monte peñascoso se compararon a las almenas de una fortaleza (DCECH, s.v. peña). Voz de uso común en toda nuestra zona de estudio con el sentido de «piedras extremadamente grandes»; [lapéna].

\subsection{Plano, cabo lo - Bacamorta}

Documentación:

a. 1623. «Die biessimo nono mensis octubris anno 1623 en el termino del lugar de Bacamorta en cabo lo plano...» (ACL, Protocolos, vol. $n .^{\circ} 13, f$. $172 r$ ).

Etimologia: en el habla viva se utiliza plano como sustantivo con el significado de «lugar llano», al igual que en otros lugares aragoneses. Apelativo que procede del latín PLANUS «llano» (DCECH, s.v. llano) con conservación del grupo PL- inicial como corresponde a la fonética altoaragonesa.

\subsection{Puyals - Valle de Terraza}

Pronunciación: [lospuyáls]

Documentación:

a.1612. «Die duo decimo mensis octobris anno 1612 et in termino de la Balle de Terraza en la partida llamada a lo puial...» (ACL, Protocolos, vol. $n^{\ell} 3, f .142 r$ ). a.1624. «...en el termino del dicho lugar de Barbaruans en la partida llamada a puials...» (ACL, Protocolos, vol. $n .^{\circ} 14, f .16 r$ ).

a.1879. «puyals» (AHPH, Am. 1215, cuad. $1 .^{\circ}$, f. 10r).

Etimología: topónimo derivado de POD ase el capítulo dedicado a la toponimia mayor, s.v. Pueyo) mediante el sufijo locativo-abundancial -al. La voz puyal no es usada generalmente como apelativo orográfico en nuestra zona de estudio, sino más bien con el significado de «montón de cereales segados». 


\subsection{Valle, la - Bacamorta}

\section{Documentación:}

a.1617. «...que tengo sitio en el termino del dicho lugar de Barcamorta en la partida llamada a la valle...» (ACL, Protocolos, vol. $n .{ }^{\circ} 8, f .153 r$ ).

Etimología: del latín VALLEM «valle», «cañada» (DCECH, s.v. vall). Voz de uso común en toda nuestra zona de estudio con el significado de «llanura de tierra entre montes o alturas»; [labál]. Compárese su género con el catalán la vall, el gascón la bat «el valle», el italiano la valle, el castellano el valle.

\section{HIDROTOPÓNIMOS}

\subsection{Canallillo - Bacamorta}

\section{Documentación:}

a.1610. «...en el termino de Bacamorta en la partida llamada a canalli1lo...» (ACL, Protocolos, vol. n. ${ }^{\circ}$ 2, f. 88r).

Etimología: topónimo derivado de canal (<CANĀLIS) (DCECH, s.v. canal) mediante el sufijo diminutivo -illo (<-ICUL(U). Creemos que la grafía $<-l l->$ medial que presenta nuestro topónimo se debe a un fenómeno de hipercorrección linguística, al ser sustituida la grafía $<\mathrm{l}>$ por la de $<\mathrm{ll}>$.

\subsection{Fonteciallas - Navarri}

Documentación:

a.1623. «...en el termino del lugar de Nabarri do dizen a fonteciallas...» (ACL, Protocolos, vol. $n .^{\circ} 13, f .28 v$ ).

Etimología: topónimo derivado de FONTE ( $\angle \mathrm{FO} N T E)$ (DCECH, s.v. fuente) mediante el sufijo diminutivo latino-ICĚLLA propio del latín imperial ${ }^{3}$ (MENÉNDEZ PIDAL. 1965:229). Su significado bien pudo ser el de «fuente pequeña».

5.3. Llagunas, lo panar de las + Foradada del Toscar Pronunciación: [lopanárdelašlagúnas] 


\section{Documentación:}

a.1613. «...en el dicho termino de Foradada en la partida llamada a lo panar de las llagunas...» (ACL, Protocolos, vol. $n .^{\circ} 4, f .203 r$ ).

Etimología: voz que procede del latín LACŪNA «hoyo, agujero» que, en principio, sirvió para denominar unas «balsas o un terreno húmedo, donde a causa de las lluvias u otros fenómenos metereológicos se posibilitaba la formación de lagunas» ( $D C E C H$, s.v. lago). Apelativo poco utilizado por las gentes de Foradada del Toscar que lo sustituyen por el sustantivo femenino paul «tierras pantanosas».

\subsection{Puzol, la binia de lo - Bacamorta}

\section{Documentación:}

a.1609. «...et asi ajuntado el dicho consejo en la binia de lo puzol...» (ACL, Protocolos, vol. $n .^{\circ} I, f .64 r$ ).

Etimología: topónimo derivado mediante el sufijo diminutivo -ol (<-OLU) de puzo. Es interesante destacar el fenómeno de metafonía que observamos en este topónimo debido a la influencia linguística que ejerce la $-U$ final átona sobre la Ú inicial tónica : PƯTĚUS > puzo; [lopú日o].

\section{5. $\operatorname{Re}(\operatorname{arcos})$ - Valle de Terraza}

\section{Documentación:}

a.1609. «...en el dicho termino del dicho lugar de la Valle de Terraza en la partida llamada a rearcos...» (ACL, Protocolos, vol. n. ${ }^{\circ}$ I, f. 196r).

Etimología: forma abreviada del sustantivo río (véase TVME I, s.v. Reperós).

\section{PROPIEDAD Y ANTROPONIMIA}

\subsection{Castelaes, serbera - Valle de Terraza}

Documentación:

a.1609. «...en el termino de la Balle de Terraza en la partida llamada a serbera castelaes...» (ACL, Protocolos, vol, $n .^{\circ} 1, f .196 r$ ).

Etimología: apelativo toponímico de difícil interpretación etimológica para el que proponemos dos posibles vías etimológicas: 
a) posiblemente se trate de un plural toponímico de castellar (<CASTELLĀRE «pequeño conjunto fortificado o territorio agregado a un castillo») ( $D C E C H$, s.v. castillo) con posterior reducción de la $-r$ - intervocálica: castellares > castellaes;

b) probablemente se trata de un apelativo toponímico derivado mediante el sufijo aumentativo -as (>-ACĒU) de castell. Sobre la forma castellas «castillo grande» se formó un plural analógico castellases con posterior reducción de la $-s$ - intervocálica: castellases $>$ castelaes.

\subsection{Enquantra, cassas de la - Valle de Terraza}

\section{Documentación:}

a.1617. «Die quinto mensis octubris anno 1617 et en las cassas de la enquantra de la Balle de Terraça» (ACL, Protocolos, vol. $n .^{\circ} 8, f .135 r$ ).

Etimología: del latín EN CŎNTRA «enfrente de» ( $D C E C H$, s.v. contra), con diptongación de la Ŏ en [wá]. Nombre de casa que debía estar situada enfrente de algún punto geográfico significativo para el núcleo de población al que pertenecía.

\subsection{Fortunno, casa de - Valle de Terraza}

Documentación:

a.1627. «Die octavo mensis januarii anno domini 1627 en la casa de Fortunno de la Balle de Terraça...» (ACL, Protocolos, vol. $n .^{\circ} 16, f .2 r$ ).

Etimología: de un antropónimo latino FORTUNIUS (SHULZE, $1991: 483)$ con palatalización del grupo $-\mathrm{NJ}->[\tilde{n}]<-n n>$.

\subsection{Gabessaco, binia de la cassa de - Foradada del Toscar}

\section{Documentación:}

a.1612. "...confronta con binia de la cassa de gabessaco...» (ACL, Protocolos, vol. $n .^{\circ} 3, f .127 r$ ).

Etimología: antropónimo para nosotros de origen incierto. La primera parte del nombre, «Gabesa-», posee un parecido lingüístico con Gavasa, núcleo de población de la Baja Ribagorza, topónimo que Corominas cree relacionado con el vasco gabe «pobre, privado de», al cual posiblemente se añadió la posposición vasca -az «con» y el artículo también vasco - $a$ (COROMINES, 1970:95). 
En cuanto a la segunda parte del topónimo, $-c o$, tal vez esté relacionada con el sufijo vasco $-k o$, que indica pertenencia, ampliamente documentado (AZKUE, 1905, s.v. $-k o$ ).

\subsection{Juan Mariniossa, la hera de - Senz}

\section{Documentación:}

a.1616. «et assi ajuntado [...] en la era de Juan Maruniossa...» (ACL, Protocolos, vol. $n .^{\circ}$ 7, f. 185r).

Etimología: Mariniossa es un apelativo muy común de la Alta Ribagorza. Corominas lo cree relacionado con una base indoeuropea y prerromana *MARONIO- derivada de un radical MAR- que se repite en el griego $\mu \alpha \pi \mu \rho o \sigma$ «bloque de piedra» (OnCat, s.v. Moruny). Si a ello añadimos que el sufijo -osa (<-ŌSA) otorga la idea de «abundancia de una cualidad en un grado supremo» fácilmente se entenderá el significado del apelativo que estamos analizando: «cantidad de bloque de piedras».

\subsection{Turlas, la cassa de - Valle de Terraza}

\section{Documentación:}

a.1614. «Die viessimo nono mensis setembris anno 1614 et en la cassa de Turlas de la Balle de Terraça...» (ACL, Protocolos, vol. $n .^{\circ} 5, f .145 r$ ).

Etimologia: apelativo que Coromines (DECat VIII, s.v. tord, $593 \mathrm{b48ss}$ ) explica a partir del cruzamiento de dos nombres de pajaros, representativos de los paisajes pirenaicos, cuyas características físicas son muy semejantes: el tord (<TŬRDUS «tordo»), [lotórd], y la merla (<MĔRŬLA «mirlo»), [lamérla]. Voz que en nuestra zona de estudio se confunde con el nombre de otra ave: la merla.

\section{PRopiedades Del TERRENo}

\subsection{Closas, las + Senz}

Pronunciación: [lasklósas]

Documentación:

a.1609. «...en el termino de dicho lugar de Senz en la partida llamada a las closas...» (ACL, Protocolos, vol. $n .^{\circ} 1, f .150 r$ ).

a.1879. «cllosas» (AHPH, Am. 1072, cuad. 1. ${ }^{\circ}, f .95 r$ ).

Etimología: procede este apelativo del latín CLAUSUS, participio del verbo latino CLAUDĚRE «cerrar» (DCECH, s.v. clausura). Se trata de una 
voz cuyo significado general en toda la Ribagorza es el «campo cerrado cercano a la casa». Ahora bien, en ciertos lugares ha dejado de ser un «campo cerrado» por lo que entra en verdadera confluencia semántica con prado. Topónimo que se aplica a un campo de cultivo cerrado ubicado en el monte Tremosvías y que pertenece a Casa Lanao.

\subsection{Llaneta, la - Bacamorta}

Documentación:

a.1618. «Die vicessimo septimo mensis dezembris anno 1618 en el termino del lugar de Bacamorta en la partida llamada a la llaneta...» (ACL, Protocolos, vol. $n .^{\circ} 9, f .1 r$ ).

Etimología: topónimo derivado en -ITTA (-eta) del celta *LANDA «lugar llano y despejado" ( $D C E C H$, s.v. landa). Corominas supone que el español landa «gran extensión de tierra llana en que sólo se crían plantas silvestres», documentado tardíamente (hacia 1800), es un préstamo del vascuence landa «campo llano, pradera» y del francés lande «landa».

Pero en la provincia de Huesca hubo de ser voz antigua, ya que si no no se encontarían tantos derivados toponímicos y además en diferentes estadios fonéticos (VÁZQUEZ, 1991:159). Por ello, parecen acertadas las palabras de Frago cuando advierte que el planteamiento de esta cuestión en el DCELC (vale lo mismo para $D C E C H$ ) no se ajusta con exactitud a la realidad histórica; en efecto, el empleo de landa como nombre de lugar no es exclusivo del dominio vasco ni del catalán, ya que la toponimia menor aragonesa es pródiga en denominaciones basadas en este término, sin que falte absolutamente en la toponimia mayor, como se demuestra en el macrotopónimo Lanuza (FRAGO, 1980:393394). De acuerdo con esto, parece lícito pensar que el área de landa en el Norte de la Península ha sido mucho más amplia de lo que generalmente se cree, y el céltico *LANDA ha podido tener entre nosostros ininterrupidamente vida desde tiempos prerromanos o, en último extremos, su procedencia galorrománica habría de retrotraerse hacia el final del siglo XI o comienzos del XII, época de masiva llegada de colonos y monjes franceses (FRAGO, 1980:122).

\subsection{Mosqueras, la cassa de las - Valle de Terraza}

Documentación:

a.1624. «Die undeçimo mensis angusti anno 1624 en el ostalet y la cassa de las mosqueras termino de la Balle de Terraça..." (ACL, Protocolos, vol. $n$. $14, f .91 v)$. 
Etimología: apelativo derivado de MŬSCA «mosca» (DCECH, s.v. mosca) mediante el sufijo -ARǏA (>-era). Voz de uso común en toda nuestra zona de estudio cuyo significado es el de «lugar sombrío donde sestea el ganado al abrigo de las moscas»; [lamoskéra].

\subsection{Perriallas, las + Senz}

Pronunciación: [lasperjálas]

Documentación:

a.1612. «...en el termino de dicho lugar de Senz en la partida llamada a las perriallas...» (ACL, Protocolos, vol. n. ${ }^{\circ} 3, f .16 v$ ).

a.1613. «...en el termino de dicho lugar de Senz en la partida llamada a las perriallas...» (ACL, Protocolos, $n .^{\circ} 3, f .54 r$ ).

Etimología: apelativo derivado mediante el sufijo diminutivo -ĚLLA (>ialla) del latín PĚTRA «piedra» (véase s.v. Insolapera) con asimilación del grupo medial latino -TR- a - $r$ - característica de nuestra zona de estudio.

\subsection{Salitre, lo - Navarri}

\section{Documentación:}

a.1610. «...un campo mio proprio sitiado en el termino del lugar de Nabarri en la partida llamada a lo salitre...» (ACL, Protocolos, vol. $n . \circ^{\circ} 2, f .5 r$ ).

Etimología: apelativo que procede del catalán salnitre (1371) formado sobre el latino NITRUM «salitre» ( $D C E C H$, s.v. sal). Voz que en nuestra zona de estudio adquiere el significado de «sustancia salina, especialmente la que aflora en tierras de cultivo»; [losalitre].

\section{SitUACIÓN Y ASPECTO DEL TERRENO}

\subsection{Cabo (lo plano) - Bacamorta}

\section{Documentación:}

a.1623. «Die biessimo nono mensis octubris anno 1623 en el termino del lugar de Bacamorta en cabo lo plano...» (ACL, Protocolos, vol. $n .^{\circ} 13, f .172 r$ ).

Etimología: del latín CAPUT «cabeza» (DCECH, s.v. cabeza) con el sentido de "punto geográfico situado en un extremo». Apelativo orográfico ampliamente conocido en toda nuestra área de estudio. 


\subsection{Corba, la + Navarri}

\section{Pronunciación: [lakórba]}

Documentación:

a.1610. «...un campo llamado a la corba...» (ACL, Protocolos, vol. $\left.n .^{\circ} 8, s / f\right)$.

a.1879. «corba» (AHPH, Am. 1072, cuad. I..$^{\circ}$ f. 30v).

Etimología: apelativo derivado del latín CŬRVUS «curvo» (DCECH, s.v. corvo). Topónimo que ha sufrido un desplazamiento semántico, ya que no se aplica a un «trozo de tierra situado en un punto geográfico curvo», sino que da nombre a un «campo de cultivo situado debajo de una gran roca cóncava que tiene forma de proa de barco».

\subsection{Cornillon - Bacamorta}

\section{Documentación:}

a.1617. «Die vigessimo nono mensis septembrisa anno domini 1627 in termino de Vacamorta in partida vocata cornillon..." (ACL, Protocolos, vol. $\left.n .^{\circ} l 6, f .55 v\right)$.

Etimología: apelativo derivado mediante el interfijo-ǏCULU- (>-ill-) y el sufijo aumentativo -ŌNE (>-ón) del latín CŎRNUS «cuerno» (DCECH, s.v. cuerno). Voz que hemos registrado en Bacamorta con el significado de «pedazuelo de tierra situada en un extremo»; [lokorniló].

\subsection{Longadera, la + Senz}

Pronunciación: [lalongadéra], [laslungadéras]

Documentación:

a. 1610. «...item otro campo sitio en dicho termino del lugar de Abi llamado la longadera...» (ACL, Protocolos, vol. $n .^{\circ} 2, f .1 r$ ).

a.1613. «...a ssaber es un campo mio proprio sittio en el termino de dicho lugar de Senz en la partida llamada a las longaderas...» (ACL, Protocolos, vol. $n{ }^{\circ} 4, f .221 v$ ).

Etimología: el apelativo longadera «campo estrecho y alargado», con conservación de la L- inicial latina, será un derivado de luengo «largo» (VÁZQUEZ, 1994:224) mediante el interfijo-ad-(>-ĀTA-) y el sufijo -era (>-ARIA), a su vez del latín LÓNGUS, -A, -UM «largo» (DCECH, s.v. largo). En Arén e Iscles, núcleos de población de la Baja Ribagorza, encontramos la partida de las Llenguaderas. 


\subsection{Solanas, las + Bacamorta \\ Pronunciación: [las:olánas] \\ Documentación:}

a.1617. «...en el termino del dicho lugar de Bacamorta en la partida llamada a las solanas...» (ACL, Protocolos, vol. $n .^{\circ} 8, f .5 v$ ).

Etimología: el apelativo solana «lugar resfuardado y donde da el sol abundantemente», al igual que solano íd., procede del latín SOLANU «viento del este», «lugar donde da el sol», derivado de SŌLE «sol» (DCECH, s.v. sol).

9. LAS VÍAS DE COMUNICACIÓN

\subsection{Arcos, re- - Valle de Terraza}

\section{Documentación:}

a.1609. «...del dicho lugar de la Valle de Terraza en la partida llamada a rearcos...» (ACL, Protocolos, vol. $n .^{\circ} l, f$. 196r).

Etimología: del latín ARCUS, -ŪS «arco» (DECH, s.v. arco). Voz característica del Valle de Bardaxín y del Valle de Lierp, aunque no de las zonas más orientales de nuestra zona de estudio, cuyo significado es el de «construcción de piedra o madera que se construye y forma sobre los ríos para poder atravesarlos». Se trata, por tanto, de un sinónimo de puente.

\subsection{Cruz (del puente biejo), la + Navarri}

\section{Pronunciación: [lakrúOdelpwénte]}

\section{Documentación:}

a. 1625. «Die decimo nonno mensis majii anno 1625 en el termino del lugar de Navarri a la cruz del puente biejo...» (ACL, Protocolos, vol. $n .^{\circ} 15, f$. $33 r)$.

Etimología: usado como apelativo, su étimo es el latín CRŬCE «cruz», «horca», «picota» (DCECH, s.v. cruz). Para la posible motivación semántica de nuestro topónimo, téngase en cuenta que en la antigüedad era práctica tradicional el amojonar o deslindar caminos y terrenos con señales en forma de cruz marcadas en las rocas o incluso con alineamientos de piedras en esa misma forma. Más rara, aunque no descartable, sería una motivación semántica en el sentido de «cruce de caminos». 


\subsection{Cruzellada, la + Navarri}

Pronunciación: [lakruӨeláda]

Documentación:

a.1609. «...un guerto mio sitiado en el lugar de Nabarri en la partida llamada a la cruzellada...» (ACL, Protocolos, vol. $n .^{\circ} 1, f .102 v$ ).

a.1617. «...que tenemos sitia en dicho lugar de Nabarri a do dizen la cruçellada...» (ACL, Protocolos, vol. $n{ }^{\circ} 8, f .132 r$ ).

a.1624. «...en el termino del lugar de Nabarri do diçen la crucellada...» (ACL, Protocolos, vol. $n .^{\circ} 14, f .158 r$ ).

Etimología: cruzellada es un derivado de CRŬCE «cruz» mediante el interfijo -ICCUL- (véase s.v. serrilla) y el sufijo locativo -ĀTA (>ada). Voz usada en todo el Valle de Lierp con el sentido de «cruce de caminos».

\subsection{Puente biejo, la cruz del + Navarri}

Pronunciación: [lakrú日 delpwénte]

Documentación:

a.1625. «Die decimo nonno mensis majii anno 1625 en el termino del lugar de Navarri a la cruz del puente biejo...» (ACL, Protocolos, vol. $n .{ }^{\circ} 15$, f. $33 r$ ).

Etimología: del latín PÓN, PONTIS «puente»(DCECH, s.v. puente) donde la $\mathrm{O}$ breve tónica latina ha resultado el diptongo -ue-. Voz de uso común en toda nuestra zona de estudio.

\subsection{Tramosvias + Senz}

\section{Pronunciación: [tremosbias]}

Documentación:

a.1608. «...en dicho luggar de Senz en la partida llamada a tramosvias...» (ACL, Protocolos, vol. $n .^{\circ} 1, f .167 v$ ).

a.1879. «tramosvias» (AHPH, Am. 1072, cuad. $1 .^{\circ}, f .95 v$ ).

Etimología: topónimo cuyo étimo es el latín ÎNTER AMBAS VǏAS «entre ambos caminos» ( $D C E C H$, s.v. entre, ambos, vía). Designa el monte común cuyo camino principal une los núcleos de población de Senz y Viu. La mayoría de las parcelas de terreno en que se divide este monte pertenecen a Casa Lanau de Senz. 
10. ZOOTOPÓNIMOS

10.1. Abelas (abellanero), lo campo de las - Foradada del Toscar

Documentación:

a.1610. «...item otro campo sitio en el mesmo termino de Foradada en la partida llamada lo campo de las abelas abellanero...» (ACL, Protocolos, vol. $n .^{\circ} 2, f .180 r$ ).

Etimología: del latín APICCULA, diminutivo de APIS «abeja» (DCECH, s.v. abeja). La evolución del grupo secunadario - K'L- a la palatal [l] es un fenómeno característico de las hablas altoaragonesas antiguas y hoy pirenaicas (FRAGO, 1987:63). Además tiene plena vigencia su derivado abellar con el sentido de "colmenar». Voz de uso común en nuestra zona de estudio; [lasabélas].

10.2. Letones, lo campo de los - Foradada del Toscar.

Documentación:

a.1613. "...que dicho campo se llama lo campo de los letones..." (ACL, Protocolos, vol. $\left.n .^{\circ} 1623, f .189 r\right)$.

Etimología: topónimo derivado de llet, del latín vulgar LACTE (<latín clásico LAC, LACTIS «leche»). En toda la Ribagorza, llitó, pronunciado [lolító], se utiliza fundamentalmente no sólo como la cría del cerdo, sino también como sinónimo de éste, sea de la edad que sea.

\subsection{Palomero, el campo de lo + Foradada del Toscar}

Pronunciación: [lokámpođelopaloméro]

Documentación:

a.1624. ...un campo mio proprio que tengo sitio en el termino de dicho lugar de Foradada vulgarmente llamado el campo del palomero...» (ACL, Protocolos, vol. $n .^{\circ} 14, f .161 r$.

Etimología: derivado del latín PALŬMBA «paloma torcaz» (DCECH, s.v. paloma) mediante el sufijo locativo-ARIU. La voz palo$m a$ es usual en todo el Valle Medio del Ésera para referirse al «ave de corral doméstica» y para designar también aquellos insectos de la familia de la mariposa. 


\subsection{Turlas, la cassa de - Valle de Terraza}

Documentación:

a.1614. «Die viessimo nono mensis setembris anno 1614 et en la cassa de turlas de la Balle de Terraça...» (ACL, Protocolos, vol. $n .{ }^{\circ}$ 5, f. 145r).

a.1623. «Eadem die et loco que jo Ciprian Palacin mancebo habitante en la cassa de turllos de la Valle de Terraca..." (ACL, Protocolos, vol. $n .^{\circ} 13, f$. $85 v)$.

Etimología: véase el apartado dedicado a la propiedad y antroponimia, s.v. Turlas, la cassa de.

\section{OTROS TOPÓNIMOS}

\subsection{Corçanes - Foradada del Toscar}

\section{Documentación:}

a.1612. «...un pedaço de campo y rengas mio sittio en el termino del lugar de Foradada [...] en la partida llamada corçanes...» (ACL, Protocolos, vol. $n .^{\circ}$ $3, f .127 r$ ).

Etimología: nombre que creemos relacionado con Corçà, nombre de dos poblaciones catalanas, mediante el sufijo de femenino plural -anes. Para Corçà, Coromines propone un étimo CURTIANU, derivado de un antropónimo latino CURTIUS (SCHULZE, 1991:78), mediante el sufijo-ĀNU (OnCat $I I$, s.v. Corçà).

\subsection{Pregoniello, lo + Espluga}

\section{Pronunciación: [lopregonjélo]}

\section{Documentación:}

a.1607. «...en la partida llamada a lo pregoniello [...] de dicho lugar despluga...» (ACL, Protocolos, vol. $n^{\circ} 1, f .90 r$ ).

Etimología: en la toponimia pirenaica catalana todavía se conserva la forma pregon y su variante preon, con pérdida de la $-g$-, con el significado de "profundo». Para este apelativo toponímico, Coromines propone un étimo latino PROFŬNDUS «profundo», el cual pasó hasta la forma actual pregon a través de pre(v)on (DECat $N$, s.v. fons, $101 \mathrm{b58ss}$ ). Topónimo que da nombre a un campo de cultivo situado en una hondonada. 


\section{Bibliograffa}

Continuamos con las mismas abreviaturas usadas en los otros artículos de esta serie. Sólo se registra aquí la bibliografía citada por primera vez.

AZKUE, M. ${ }^{a}$ ResURRECCIÓN (1905): Diccionario Vasco-español-francés, Bilbao.

COROMINES, JoAN (1970): Estudis de toponimia catalana II, Barcelona, Biblioteca Fi-

lolòliga Barcino, p. 95.

Frago García, Juan Antonio (1980): Toponimia del Campo de Borja, Zaragoza, Institución «Fernando El Católico», p. 122 y pp. 393-394.

- (1987): "Toponimia navarroaragonesa del Ebro (VI): fauna", Archivo de Filología Aragonesa, XXXIX, p. 63.

MenÉndez PIDAl. RAMÓN (1965): Manual de gramática histórica española, Madrid, Espasa-Calpe, p. 229.

TVMEI = SELFA SASTRE. MOISÉs, «Toponimia documental del Valle Medio del Ésera (I)», Alazet, 10 (1998), pp. 181-203.

VÁzQUEZ OBRADOR. JEsús (1994): «Para un corpus de toponimia tensina II: registros en protocolos notariales de los años 1478-1483", Alazet, 6 (1994), pp. 203-241. 